\title{
Measurements and Simulations to Evaluate Strategies for Improved Energy Efficiency of a Reach Stacker, Forwarder and Wheel Loader
}

\author{
Olof Noréus , Magnus Hägglund, and Mats Emlén \\ ITH, Institute of Applied Hydraulics, Örnsköldsvik, Sweden \\ E-mail: ons@ith.se
}

\begin{abstract}
As the boom on for example a conventional reach stacker, a forwarder or a wheel loader is retracted and lowered, the entire potential energy of the boom and the load is wasted and converted into fluid heat in the control valves. To reduce the energy consumption and power installation requirements, one possible solution is to compensate for the empty weight, for example by utilising a spring or gas accumulator. By integrating a gas accumulator into the piston rod of the boom cylinder, it is possible to improve the energy efficiency on existing equipment just by changing the boom cylinder. This solution can be used in conventional hydraulic systems but it also has potential to reduce power consumption in more complex electric or hydraulic potential energy recuperation solutions.

A number of load handling sequences have been simulated in SimulationX software using a conventional hydraulic reach stacker load handling simulation model and simulation results have been compared to measurements. The simulation results show a good overall compliance with reality. The simulation model can provide detailed information about how large the losses are in different components of the system. By modifying the model and utilise the measured data to get a relevant loading cycle, it is possible to evaluate strategies for improved energy efficiency. Performed simulations indicate that it is possible to further improve the energy efficiency of the system by adding a cylinder and accumulator to recuperate energy when lowering the boom. That also enables the possibility to downsize and reduce the cost for hybrid solutions.
\end{abstract}

Keywords: Energy Efficient Hydraulics, Mobile Load Handling, Crane, Reach Stacker

\section{Introduction}

Hydraulic actuators are commonly used in mobile load handling systems due to large benefits such as high power density, large force and torque capability and robustness regarding chock loads. In mobile systems it is important to keep the weight down and the hydraulic system is often kept rather simple both to reduce weight and cost.

Although such hydraulic systems are efficient regarding weight and force or torque performance, they tend to be less impressive when it comes to energy efficiency. For systems where the hydraulic actuators only are used a small part of the time, that might not be an issue, since keeping the weight and cost down keeps the complete system efficient.

However, with increased energy costs the motivation to improve the energy efficiency also increases. In particular in applications where the hydraulic actuators are heavily used, it is beneficial to make the hydraulic system more energy efficient.

Methods to improve the energy efficiency are discussed in Section 2. A common energy loss in load handling systems is that energy is consumed to lift the empty weight, and that energy is then dissipated as the boom is lowered.

\subsection{Evaluated applications for energy saving}

Measurements have been performed on a wheel loader, a reach stacker and a forwarder. Each vehicle is equipped with a hydraulically manoeuvred boom.

The focus in this study is to include a support cylinder that recuperates potential energy from the empty weight when lowering the boom, and then contributes to the energy needed to lifting the empty weight when raising the boom. Such a cylinder is described in Section 2.1 and the application to a wheel loader is discussed as an example. The boom lift cylinder pressure for a conventional cylinder has been measured during full stroke loading cycles. The energy saving potential, when compensating for the empty weight, is estimated by calculations.

Measurements and modelling of a reach stacker to make it possible to analyse the energy saving potential in a simulated environment are described in Section 3 and 4. Pressure and flow have been measured at several positions in the system 


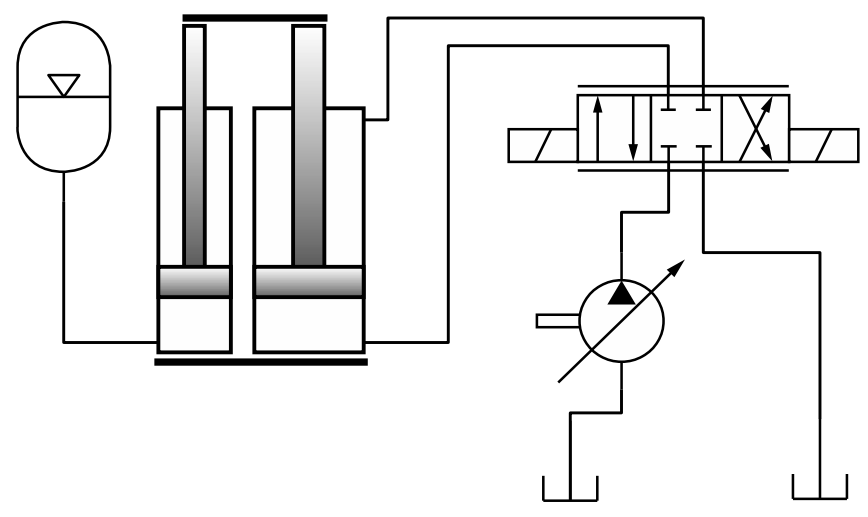

Figure 1: Addition of support cylinder compensating the empty weight for improved energy efficiency.

while using conventional lift cylinders and a dynamic model is validated by comparing simulation results to the measurements. The energy saving potential is estimated by including energy saving cylinders in the model and performing dynamic simulations. Using a dynamic model of the complete system also makes it possible to analyse effects on other parts of the system and potential problems such as oscillations.

Finally, the energy saving potential by applying energy efficient cylinders on the reach stacker and a forwarder, estimated by dynamic simulations, are presented in Section 5. For the forwarder, measurements of lift cylinder pressures and position have been made and a simplified dynamic model, including the lift cylinder and the load, is utilised to estimate the energy saving potential.

\section{Strategies for improving energy efficiency}

The hydraulic system in, for example, the Kalmar DRF450 reach stacker contains energy saving control strategies. The variable pump is controlled using a load sensing signal from the directional control valve in order not to provide more pressure than necessary from the pump. In addition, the regenerative valves enable faster lifting and reduced pump flow demand at moderate loads by leading the oil from the cylinder minus side through a spring biased check valve to the cylinder plus side.

There are several possible strategies to further improve the energy efficiency, e.g. described in [1,2]. One evaluated example is to add a cylinder and accumulator, according to fig. 1, that acts like a spring and recuperates energy when lowering load and contributes to the force when lifting.

\subsection{Energy efficient cylinder with gas accumulator}

Instead of adding another cylinder with accumulator to compensate for the empty weight as in fig. 1, a solution developed by THORDAB AB is to integrate the accumulator into a cylinder by using a hollow piston rod, as shown in fig. 2 . The piston rod is filled with gas and an accumulator piston rod is added inside it. Thus, the piston rod is also acting as a cylinder filled with gas.

Depending on the piston rod length, it may be possible to have

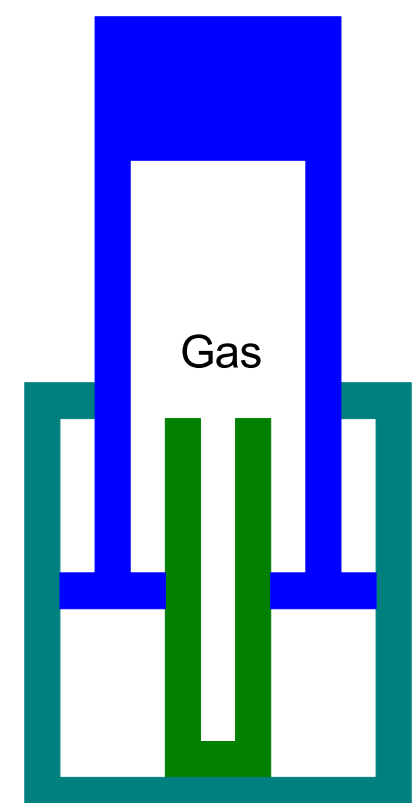

Figure 2: Energy efficient cylinder with integrated gas accumulator developed by THORDAB AB.

a gas volume in the top of the piston rod, above the accumulator piston rod stroke, to ensure that the compressed gas volume is large enough when the accumulator piston rod is pushed fully into the piston rod. Additionally, by letting the seals be mounted inside the piston on the piston rod and using a hollow accumulator piston rod, an additional gas volume is created. Thereby, compressing the gas too much can be avoided.

Both single acting and double acting cylinders are utilised in load handling systems. For equipment with single acting cylinders, it is necessary to not let the accumulator piston rod force exceed the empty weight in order to be able to lower the boom.

When double acting cylinders are used, it may be possible to even out the power demand during the loading cycle by letting the accumulator piston rod provide a force that is higher than the empty weight. It will then be necessary to actively push the boom down, i.e. it is necessary to provide hydraulic power also when lowering the boom without load, but the power demand during the lifting sequence is further reduced.

Depending on the maximum allowable gas pressure and the desired force from the accumulator piston rod, it is possible to determine an appropriate accumulator piston rod outer diameter. In order to eliminate the need to fasten the accumulator piston rod in the cylinder bottom, it is beneficial to never let the gas pressure drop below the hydraulic pressure. To maintain enough gas pressure during the cylinder stroke, a large enough gas volume is needed in the accumulator.

If the accumulator should be fully integrated within the cylinder, that implies that a minimum inner diameter of the piston rod can be determined. In fig. 3, the accumulator piston rod diameter and piston rod inner diameter are shown for varying maximum gas pressures. 


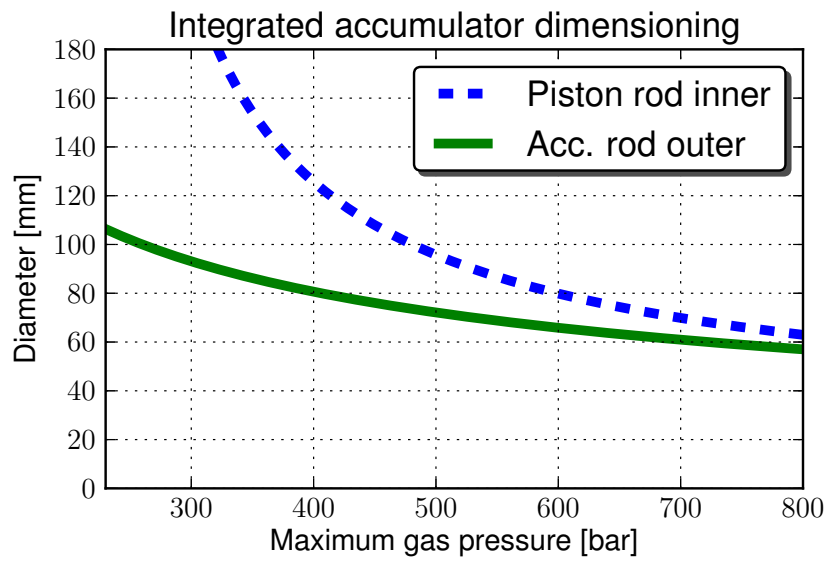

Figure 3: Dimensioning of integrated accumulator in cylinder. The accumulator piston rod outer diameter and the piston rod inner diameter are depending on the maximum desired gas pressure.

The cylinder in this example is appropriate for the Atlas Copco ST14 mining wheel loader. The cylinder is double acting and the accumulator piston rod is chosen such that it compensates for the empty weight, while the piston rod inner diameter is chosen such that the gas pressure never drops below the hydraulic pressure.

Assuming that the normal compressed gas pressure should be 350 bar, fig. 3 indicates that the appropriate outer diameter of the accumulator piston rod is about $85 \mathrm{~mm}$ and the corresponding piston rod inner diameter is about $155 \mathrm{~mm}$.

With the chosen accumulator design, the gas pressure is shown in fig. 4 . In fig. 5 the measured hydraulic pressure in a standard cylinder when lifting and the corresponding estimated pressure in the accumulator cylinder are shown. Comparing to fig. 4 , it can be seen that the hydraulic pressure never exceeds the gas pressure.

In this application the hydraulic pressure increases with the cylinder stroke. Since the gas pressure drops as the gas is expanding, the accumulator will support a larger amount of the load at lower positions. Therefore, the hydraulic pressure for the accumulator cylinder approaches the pressure of the standard cylinder as the stroke position increases. Ideally, the hydraulic pressure demand would be decreasing with the stroke position, allowing the gas pressure to support about the same amount of the load at all stroke positions.

However, although the pressure demand approaches the demand for the standard cylinder at full stroke, the accumulator piston rod reduces the volume in the cylinder. Therefore, the flow to the cylinder is reduced at all positions and thereby also the power demand, which is shown in fig. 6.

The reduced pressure demand for the accumulator cylinder shown in fig. 5 contributes to the reduced hydraulic power consumption for the cylinder, but the global effect on the complete system varies with the configuration.

If the pump pressure cannot be reduced, for example due to other consumers demanding a high pressure in an LS sys-

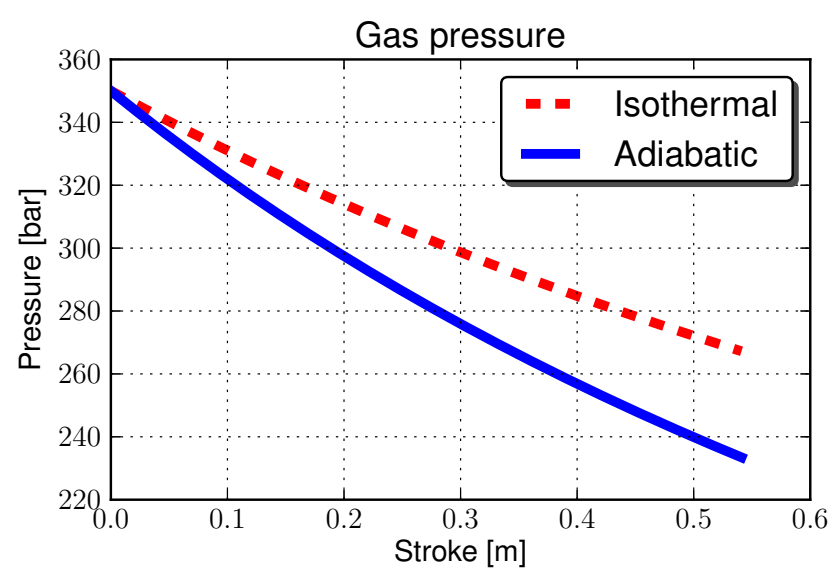

Figure 4: The gas pressure decreases when lifting.

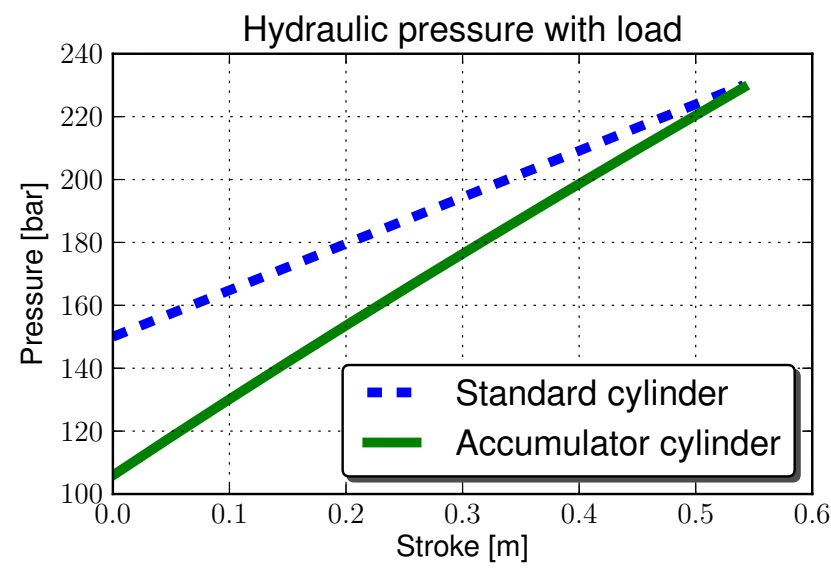

Figure 5: Hydraulic pressure in the cylinder when lifting. The pressure in the standard cylinder is measured during a real loading cycle, while the corresponding pressure for the accumulator cylinder is estimated.

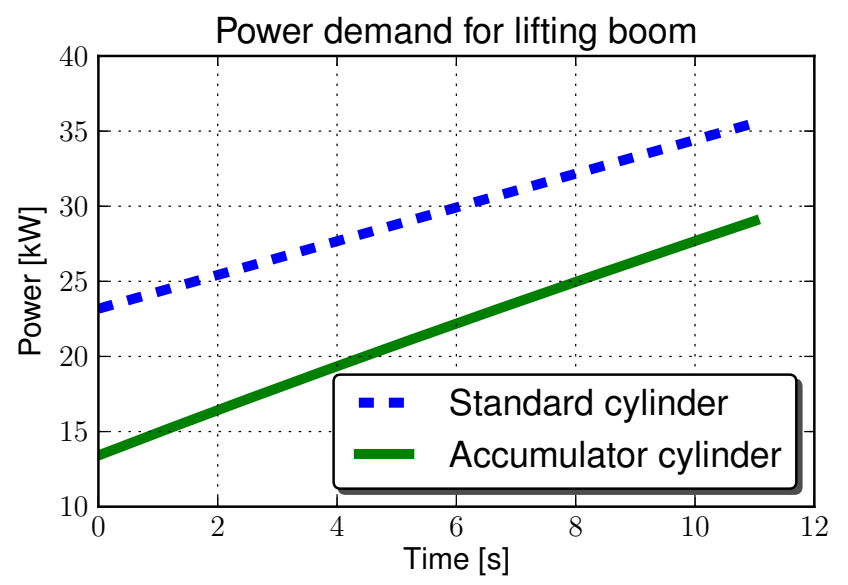

Figure 6: Power demand for each cylinder when lifting the boom, estimated from measured pressure on the standard cylinder, neglecting losses. 
tem (described in Section 4.1), the control valve losses are increased instead. Therefore, the reduced pressure demand does not necessarily contribute to a reduced power consumption for the complete system.

On the other hand, if the standard cylinder was the consumer demanding the highest pressure, changing it to an energy efficient cylinder demanding a lower pressure would reduce valve pressure losses also for other consumers. Moreover, the decreased flow demand not only reduces the cylinder power, but also reduces pressure drop losses in the system, regardless of other consumer's demands.

From an energy point of view it is beneficial to increase the gas volume in this case, since the gas pressure is better maintained during the stroke. If it is not possible to increase the piston rod inner diameter, it may be possible to add an external gas bottle.

The maximum possible energy saving would in this case be if the gas pressure could be kept constant during the stroke. In that case $110 \mathrm{~kJ}$ per cylinder and stroke would be saved of the total $320 \mathrm{~kJ}$ needed from each cylinder to lift the boom. That is, it is not possible to save more than $34 \%$ of the energy when lifting with an accumulator cylinder in this application.

For the accumulator integrated within the cylinder, the reduced power demand shown in fig. 6 corresponds to an energy saving of $88 \mathrm{~kJ}$, corresponding to $27 \%$ for the cylinder, not including losses and effects on the rest of the system.

\section{Measurements on a reach stacker}

A reach stacker is a vehicle used for handling cargo containers and are able to quickly transport containers short distances and pile them. When lowering heavy containers, there is a great energy saving possibility, since the potential energy usually is dissipated as heat.

The reach stacker considered in this study is a Kalmar DRF450 reach stacker manufactured by Cargotec and shown in fig. 7. It handles 20 and 40 feet containers with a maximum lift capacity of approximately $45 \mathrm{t}$.

Measurements have been carried out when lifting the container to full height. All sequences have been made with $0 \mathrm{t}, 27 \mathrm{t}$ and $45 \mathrm{t}$ containers resulting in a range of different load handling sequences. In addition, these sequences have all been carried out both for fully and slowly increasing joystick actuation at full engine speed. Also, all measurements were repeated with two flow turbines measuring both pump flows.

Measuring pump speed, flow, pressures in the system and boom angle and extension enables verification of the simulation model for the unmodified system and also gives a relevant driving cycle to use in the simulations.

\section{Modelling of a reach stacker}

A 3D multibody system (MBS) model as well as a load handling hydraulic model of a conventional reach stacker container truck has been built using the SimulationX software, described for example in [3-5].

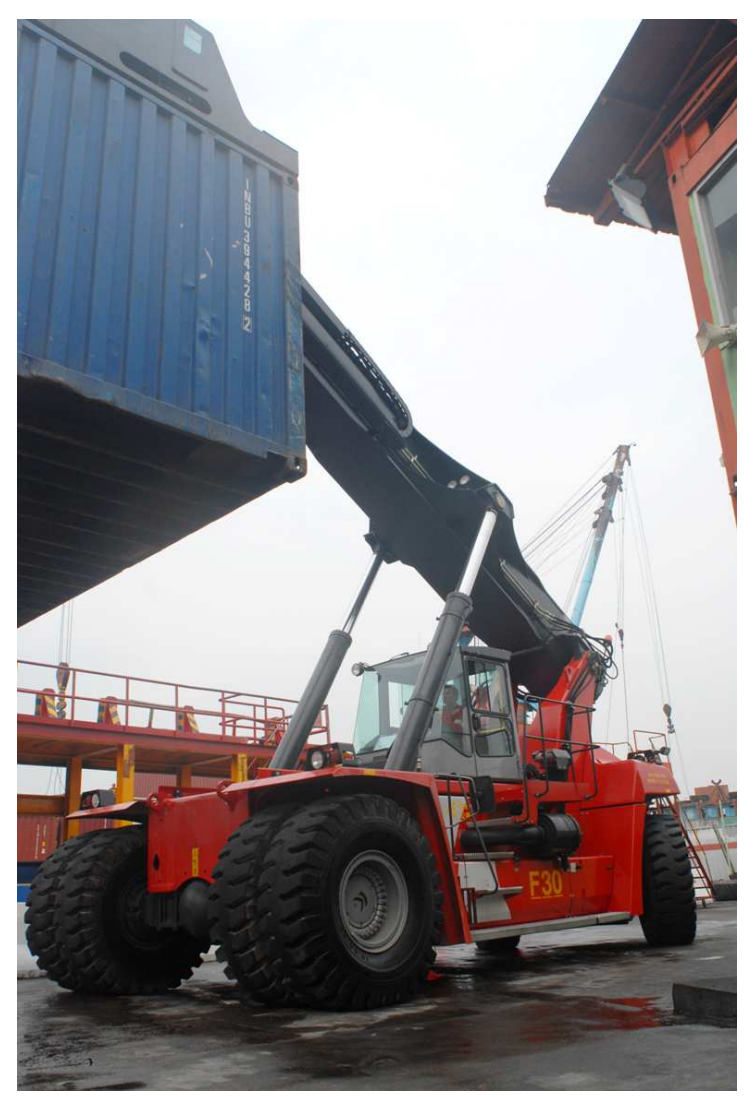

Figure 7: Kalmar reach stacker.

\subsection{Hydraulic model}

The hydraulic system, shown in fig. 8, managing the boom lift and boom extension functions of the reach stacker is a so called load sensing (LS) system. This means that the displacements of the variable pumps are controlled in order to match the highest pressure demand. The simulation model consists of standard SimulationX element types such as variable pumps, differential cylinders and valves, as well as a number of element types specially assembled for this system: the M402 directional control valve, the variable pump control block and the regenerative valve block.

For the hydraulic cylinders, a mechanical efficiency of $97 \%$ is assumed. Moreover, they are simulated with a rigid end stop and no internal or external leakages are considered.

In addition to the hydraulic components, there is also a boom assembly multibody system (MBS) connected to lift and extension cylinders.

\subsection{Mechanical model}

The mechanical model is divided into two submodels; one load handling submodel and one running gear submodel. This way load handling can be simulated separately by the load handling submodel or both load handling and driving can be simulated by connecting the two submodels to each other. It is important that the submodels can be connected when evaluating system solutions where energy is transferred from one subsystem to another. The simulation tool is considered to 


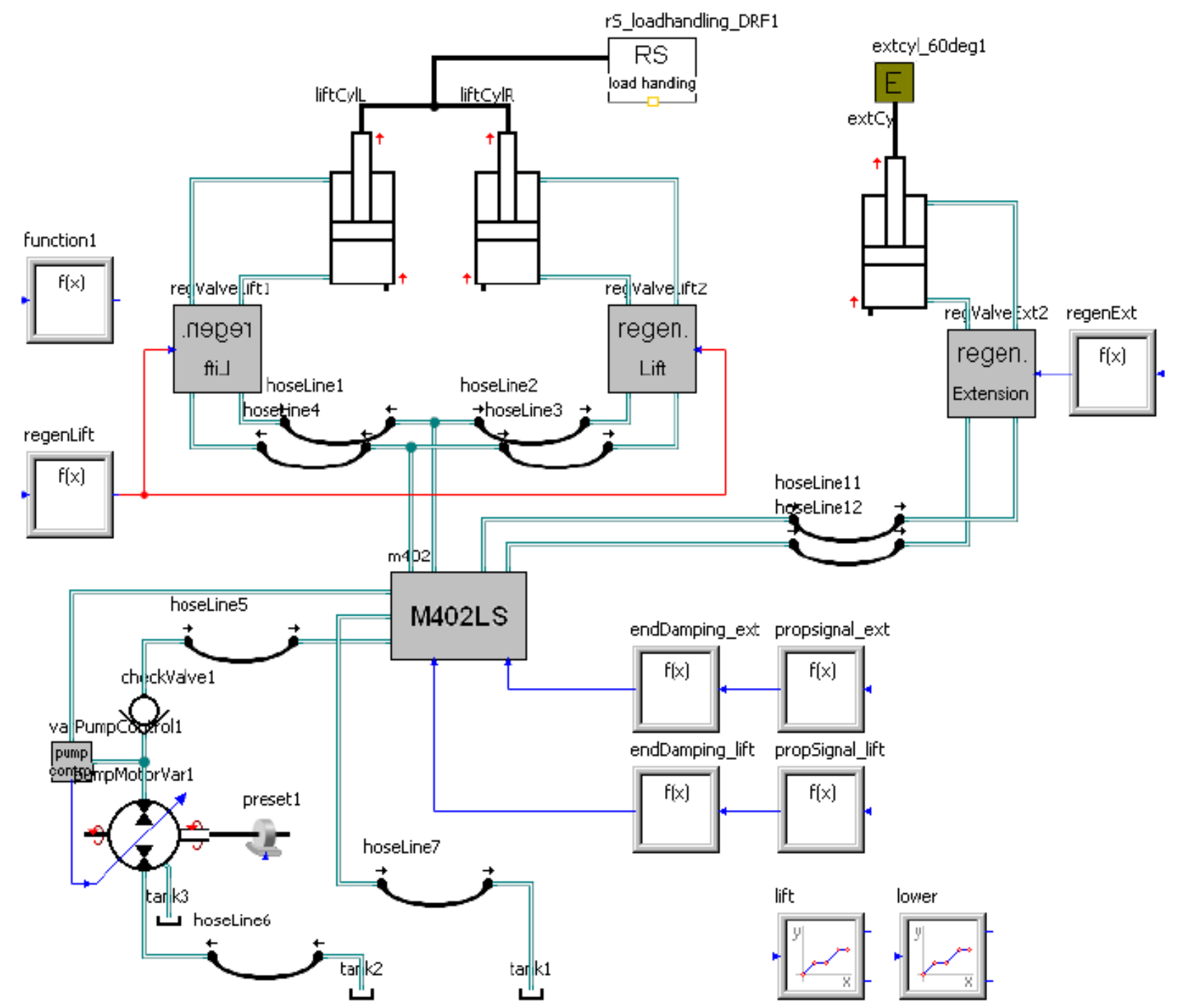

Figure 8: Simplified hydraulic model in SimulationX.

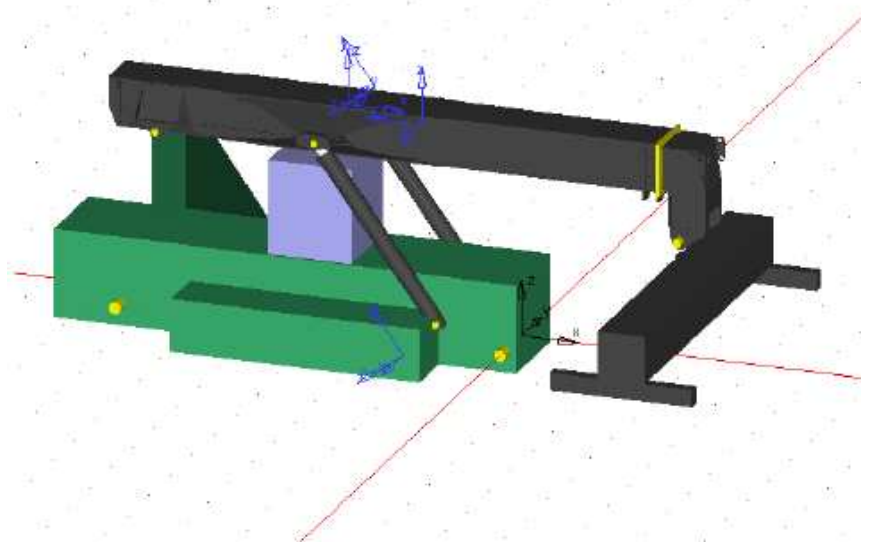

Figure 9: Load handling visualisation of simulation model. be efficient if the whole system as well as subsystems can be effectively studied. The two submodels are built by rigid bodies, joints and forces from the SimulationX 'MBS Mechanics' library and tire elements from the 'Power Transmission MBS' library.

\subsubsection{Load handling model}

The load handling model, shown in fig. 9, consists of a number of rigid bodies representing chassis, outer boom, inner boom, spreader, container etc.; each of them with a defined geometry and mass. In addition it has a lift cylinder force, a boom rotational joint, a boom extension joint and a spreader rotational joint.

The boom can be set into lift and extension motion by affecting the lift cylinder force and the boom extension joint by mechanical forces. During simulation, these forces can be determined for example by simply defining a velocity or by connecting a hydraulic cylinder from a hydraulic simulation model.

The rotational and prismatic joints of the lift cylinders are assumed to be frictionless. Rigid friction with continuous 
transition is considered for the boom rotational and extension joints as well as for the spreader joint, with the coefficient of slipping friction set to 0.1 , the limit angular and linear velocity difference set to $5 \times 10^{-5} \mathrm{rad} / \mathrm{s}$ and $5 \times 10^{-5} \mathrm{~m} / \mathrm{s}$, respectively, and the friction radius set to $100 \mathrm{~mm}$.

\subsubsection{Vehicle running gear model}

The driving model consists of rigid bodies with geometries and mass representing rims and tires. It also includes 'Tire Plane Contact' elements simulating the tire motions, a force element representing the drag force and rotational as well as translational joints in order to enable the driving motions of the vehicle. The vehicle is set into motion by affecting the left and right front tires by mechanical torques. During simulation, the torques can be determined for example by simply defining an angular velocity or by connecting a differential gearbox from a drive system simulation model.

\section{Results}

The measurements provide both relevant load cycles and data for verification of models. By using the simulation model, it is possible to identify where in the system most energy is dissipated and evaluate possible energy savings by modifying the system.

\subsection{Verification of models}

The reach stacker load handling system simulation model's compliance with reality has been evaluated by comparing simulation results with measurement data for different load handling sequences. The evaluation shows a good overall compliance.

\subsection{Evaluation of energy losses}

Simulating the system according to the driving cycle makes it possible to identify the components that contribute most to the energy losses. Thereby it is clarified what parts in the system it would be most beneficial to further develop in order to achieve an improved energy efficiency.

\subsection{Improved energy efficiency}

When using an accumulator to recuperate energy, the amount of saved energy is depending on both the gas prefill pressure and the initial oil pressure in the accumulator. When lifting a $27 \mathrm{t}$ container, it is reasonable to save about $10 \%$ to $30 \%$, which is shown in fig. 10 and fig. 11 . This is based on simulations of the lifting phase, i.e. the energy saving is not representative for a complete loading cycle.

\subsection{Energy efficient cylinder applied to a forwarder}

A forwarder is a forest machine equipped with a crane and grasper used for loading and transporting logs.

A similar energy efficiency simulation, studying the effects of adding an extra cylinder, as described in the reach stacker case was also performed on a forwarder. In this case an El-Forest forwarder with a Cranab FC crane was continuously lifting and lowering the boom without load in the grasper, as shown

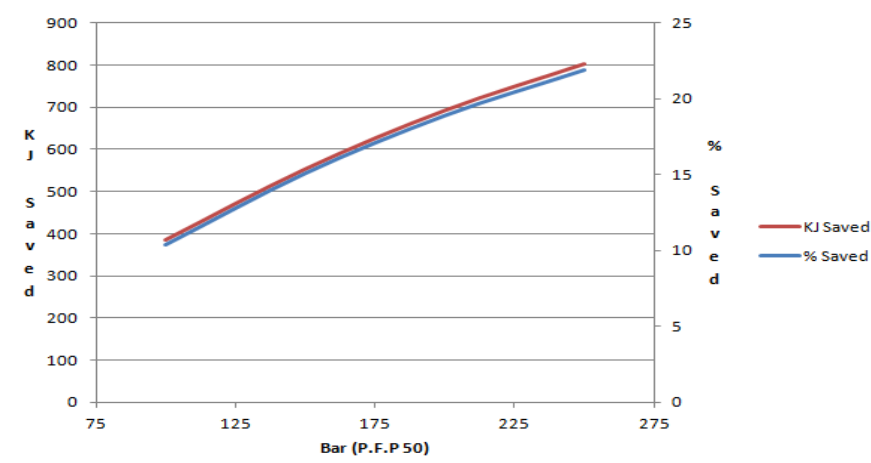

Figure 10: Energy saving vs. initial oil pressure when lifting a $27 t$ container. The prefill pressure in the accumulator is 50 bar.

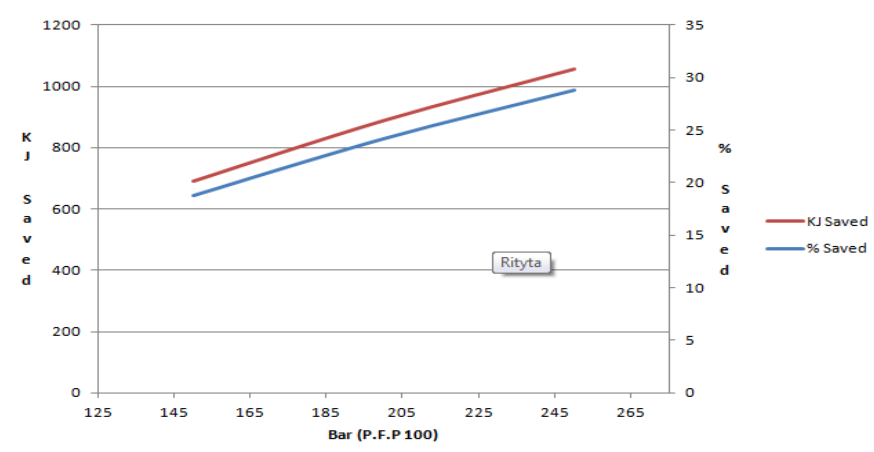

Figure 11: Energy saving vs. initial oil pressure when lifting a $27 t$ container. The prefill pressure in the accumulator is $100 \mathrm{bar}$.

in fig. 12 and 13. During this time the lift cylinder pressure and position were measured. These measurements were used as input in the simulation.

The model is simpler than in the reach stacker case only focusing on the lift hydraulics. The simulation is supposed to follow the measured lift cylinder motion. Therefore a constant pressure pump is used with a PID controlled proportional direction valve to the lift cylinder. The controller setpoints are the measured positions of the cylinder.

The force acting on the cylinder was calculated from the measured pressure. The lift cylinder pressure and motion in the simulation correspond to the measured values, which validates the model. The hydraulic power in the lift cylinder from simulations with and without an additional cylinder and gas accumulator was compared.

The results indicate an improved energy efficiency of about $20 \%$ during lifting, using the additional cylinder. The pressure could be lowered compared to a conventional cylinder. As the pump pressure in an LS system adjusts to the highest pressure demand this could mean less energy loss in pressure drop to other functions during normal driving cycles. Problems during simulation were oscillations, which may or may not occur in reality. 


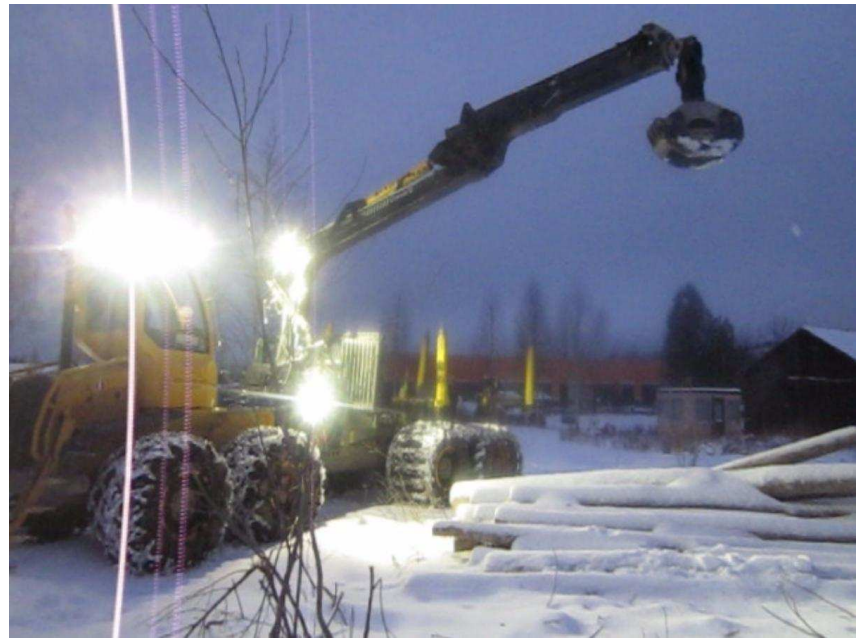

Figure 12: Forwarder with boom in high position without load.

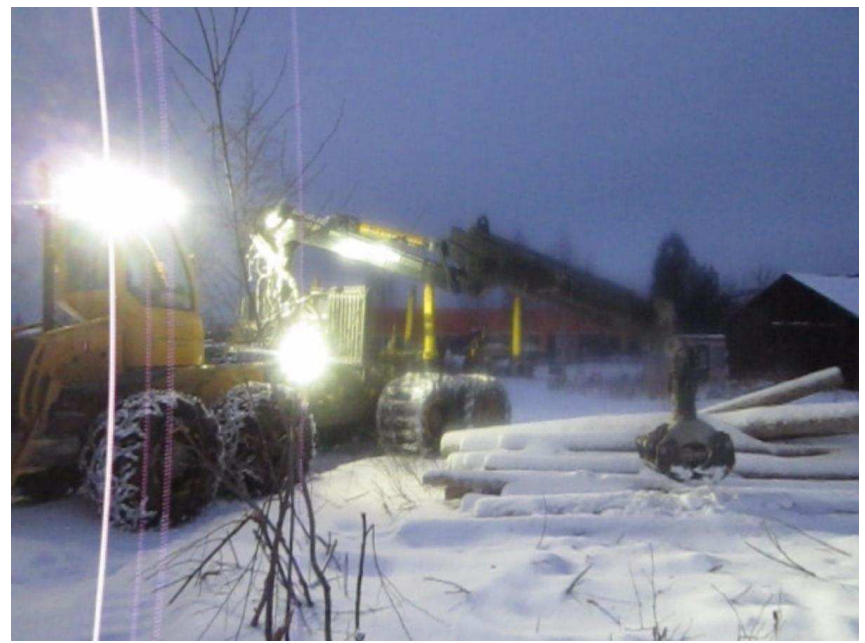

Figure 13: Forwarder with boom in low position without load.

\section{Conclusions}

A validated MBS heavy equipment model can be a very useful tool not only for evaluating energy efficiency but also for a number of additional design work question formulations, in both new design and design modifications. Axle pressure figures could be used for determining the vehicle stability in extreme load positions and/or motions. Power figures could be used for dimensioning hydraulic and propulsion systems out of a vehicle performance specification. MBS simulations are of great value in early design phase as well as a tool for simulation driven design.

The performed simulations and calculations applied to a reach stacker, forwarder and wheel loader indicate that it is possible to further improve the energy efficiency of mobile load handling systems by adding a cylinder and accumulator to recuperate energy when lowering the boom. Since the cylinder reduces the power and energy demand, it also has the potential to reduce the cost and improve the efficiency of more complex electric or hybrid energy recuperating systems. Losses will decrease as less power has to be handled by for example a battery. Moreover, smaller components can be used.

One obstacle when developing electric or hybrid energy recuperating systems might be the increased initial cost for the system. Therefore, the energy efficient cylinder can not only replace a conventional cylinder to improve the efficiency of existing equipment, but it can also enable the development of electric or hybrid energy recuperating systems by reducing the power demand and thereby also the initial cost.

\section{References}

[1] Xingui Liang and Tapio Virvalo. Energy reutilization and balance analysis. Proceedings of the Fifth International Conference on Fluid Power Transmission and Control (ICFP), 4:306-310, 2001.

[2] Karl-Erik Rydberg. Energy efficient hydraulic systems and regenerative capabilities. Proceedings of the Ninth Scandinavian International Conference on Fluid Power, SICFP'05, 2005.

[3] Hans Dresig and Franz Holzweißig. Dynamics of Machinery: Theory and Applications. Springer-Verlag, Berlin Heidelberg, 2010. ISBN 978-3-540-89939-6.

[4] Saeed B. Niku. Introduction to Robotics: Analysis, Control, Applications. John Wiley \& Sons, 2010. ISBN 9780-470-60446-5.

[5] Ryszard Jablonski, Mateusz Turkowski, and Roman Szewczyk. Recent Advances in Mechatronics. SpringerVerlag, Berlin Heidelberg, 2007. ISBN 978-3-54073955-5. 